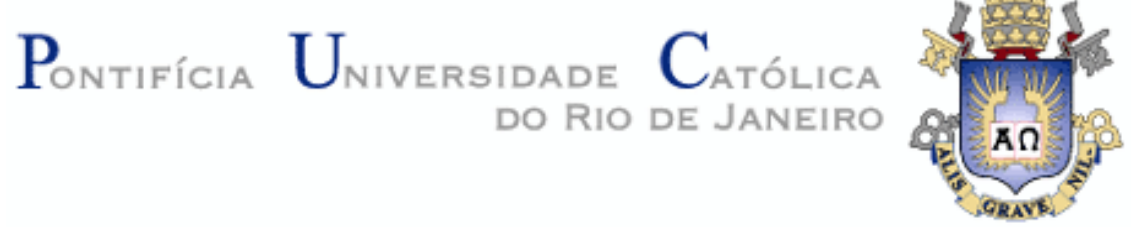

Carolina Caldas do Nascimento

\title{
O valor da opção do carro Flex por região geográfica do Brasil: uma aplicação do TOR com MRM
}

\begin{abstract}
Dissertação de Mestrado
Dissertação apresentada como requisito parcial para obtenção do grau de Mestre pelo Programa de PósGraduação em Engenharia de Produção do Departamento de Engenharia Industrial da PUC-Rio.
\end{abstract}

Orientador: Prof. Carlos Patrício Samanez Coorientador: Prof. Marco Antônio Guimarães Dias

Rio de Janeiro Janeiro de 2012 


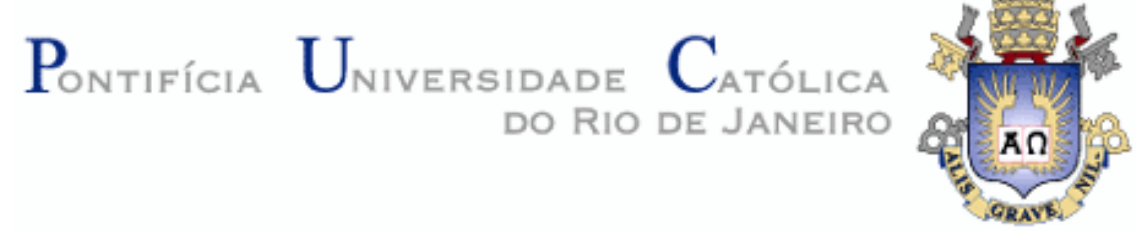

Carolina Caldas do Nascimento

\begin{abstract}
O valor da opção do carro Flex por região geográfica do Brasil: uma aplicação do TOR com MRM
\end{abstract}

Dissertação apresentada como requisito parcial para obtenção do grau de Mestre pelo Programa de Pós-Graduação em Engenharia de Produção do Departamento de Engenharia Industrial da PUC-Rio. Aprovada pela Comissão Examinadora abaixo assinada.

Prof. Carlos Patrício Samanez Orientador Departamento de Engenharia Industrial - PUC-Rio

Prof. Marco Antonio Guimarães Dias Co-orientador Departamento de Engenharia Industrial - PUC-Rio

Prof. José Francisco Moreira Pessanha Departamento de Matemática e Estatística - UERJ

Prof. Paulo Henrique Soto Costa Departamento de Engenharia Industrial - PUC-Rio

Prof. José Eugenio Leal Coordenador Setorial do Centro Técnico Científico - PUC-Rio 
Todos os direitos reservados. É proibida a reprodução total ou parcial do trabalho sem autorização da autora, do orientador e da universidade.

\section{Carolina Caldas do Nascimento}

Graduou-se em Estatística pela UERJ em julho de 2008. Foi analista de Preços e Tarifas da Empresa Brasileira de Telecomunicações - EMBRATEL. Seus interesses de pesquisa são avaliação de investimentos e planejamento estratégico.

\section{Ficha Catalográfica}

Nascimento, Carolina Caldas do

O valor da opção do carro Flex por região geográfica do Brasil: uma aplicação do TOR com MRM / Carolina Caldas do Nascimento ; orientador: Carlos Patrício Samarez; co-orientador: Marco Antônio Guimarães Dias - 2012.

117 f. : il. (color.) ; $30 \mathrm{~cm}$

$$
\text { Dissertação (mestrado)-Pontifícia }
$$

Universidade Católica do Rio de Janeiro, Departamento de Engenharia Industrial, 2012.

Inclui Referências Bibliográficas

1. Engenharia Industrial - Teses. 2. Opções reais. 3. Processos estocásticos. 4. Carro Flex-fuel. 5. Reversão à média. 6. Simulação de Monte Carlo.

7. Etanol. I. Samarez, Carlos Patrício. II.Dias, Marco Antônio Guimarães. III. Pontifícia Universidade Católica do Rio de Janeiro. Departamento de Engenharia Industrial. IV. Título. 
Para os meus pais e meu noivo, pelo apoio e confiança 


\section{Agradecimentos}

Ao meu orientador e coorientador, Prof. Carlos P. Samanez e Prof. Marco Antônio G. Dias, pelo estímulo e contribuições nesse estudo.

A CAPES e à PUC-Rio, pelos auxílios concedidos, sem os quais este trabalho não poderia ter sido realizado.

Ao meu noivo, Peter Heinroth, por todo incentivo, paciência e dedicação.

Aos meus pais, pela educação, investimento e confiança durante os meus estudos.

Aos professores do Departamento de Eng. Industrial, pelos ensinamentos e ajuda durante todo meu período de mestrado.

Aos professores que participaram da Comissão examinadora, por contribuírem com ideias e sugestões para esse estudo.

A secretaria do Departamento de Eng. Industrial da PUC-Rio, especialmente a Cláudia, sempre disposta a ajudar e resolver dúvidas.

Ao Prof. José Franscisco do Departamento de Matemática e Estatística da UERJ, por sempre motivar seus alunos a terem sede de conhecimento. 
Aos meus amigos da vida, faculdade e Embratel por motivarem o investimento nesse estudo e acreditarem nesse sonho. 


\section{Resumo}

Nascimento, Carolina Caldas; Samanez, Carlos Patrício; Dias, Marco Antônio Guimarães. O Valor da Opção do Carro Flex por região geográfica do Brasil: uma aplicação do TOR com MRM. Rio de Janeiro, 2012. 117p. Dissertação de Mestrado - Departamento de Engenharia Industrial, Pontifícia Universidade Católica do Rio de Janeiro.

A introdução do carro Flex-fuel no mercado brasileiro em 2003 mudou a forma de decisão do consumidor. Se antes era necessário escolher o combustível pelo tipo de veículo, movido somente a gasolina ou somente a álcool, hoje é possível escolher um tipo de carro com duas opções de combustível. O carro Flex funciona com gasolina, etanol ou qualquer mistura desses combustíveis em qualquer proporção. Essa flexibilidade gera uma vantagem econômica para o seu proprietário, mas qual o benefício financeiro de um carro Flex-fuel em comparação a um carro movido somente à gasolina? Geograficamente, onde se localiza o proprietário que se beneficia mais dessa flexibilidade? Este estudo aplica a Teoria de Opções Reais na análise do valor da opção do carro Flex para as cinco regiões geográficas do Brasil: Nordeste, Norte, Centro-Oeste, Sudeste e Sul. São atendidas tanto as diferenças de preços regionais quanto a preferência de automóvel do consumidor dessas regiões. Para esse propósito, são considerados que os preços históricos dos combustíveis são estocásticos e seguem o Movimento de Reversão à Média Aritmético. A previsão dos preços e o valor da opção são gerados através da Simulação de Monte Carlo. Os resultados indicam que a opção de escolher o combustível mais barato no abastecimento adiciona considerável valor para o proprietário do carro Flex em todas as regiões e modelos de carro considerados, sendo a região Sudeste a mais beneficiada pela opção Flex.

\section{Palavras-chave}

Opções Reais; Processos Estocásticos; Carro Flex-fuel; Reversão à Média; Simulação de Monte Carlo; Etanol. 


\section{Abstract}

Nascimento, Carolina Caldas; Samanez, Carlos Patrício (Advisor); Dias, Marco Antônio Guimarães (Co-advisor). The option value of the Flex-fuel car in different geographic regions of Brazil: application of Real Options Theory with MRM. Rio de Janeiro, 2012. 117p. MSc. Dissertation - Departamento de Engenharia Industrial, Pontifícia Universidade Católica do Rio de Janeiro.

The introduction of the Flex-fuel car to the Brazilian market in 2003 changed the way of decision of the customers. If before it was necessary to choose the fuel by the kind of vehicle, fueled only by gas or only by sugarcane ethanol, today it is possible to choose one kind of car with two fuel possibilities. The Flexfuel car can run with gas, sugarcane ethanol or any mix of those fuels at any proportion. This resulting flexibility generates an economic advantage for the car's owners, but what are the finance benefits of having a Flex-fuel car in comparison to a gas-powered car? Where is geographically located the owner which benefits more from this flexibility? This study applies the Real Option method to analyze the option value of the Flex-fuel cars in each of the five geographic regions of Brazil: Northeast, North, Central-West, Southeast and South. It regards regional prices differences and their customer's car preference. For this purpose, it considers that the historical fuel prices are stochastic and follow the Arithmetic Mean Reverting diffusion process. Monte Carlo Simulation provides the forecasted fuel prices and option values. The results show that the option to choose the cheapest fuel at the refueling moment adds significant value for the owner of all the regions and car models considered. The Southeast region is the most benefited from it.

\section{Keywords}

Real Options; Stochastic Process; Flex-fuel car; Mean Reversion Process; Monte Carlo Simulation; Ethanol. 


\section{Sumário}

1 Introdução................................................................................................ 16

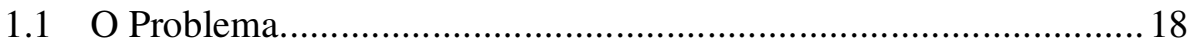

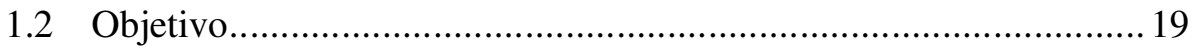

1.3 Posicionamento da dissertação e Estado da Arte ...............................20

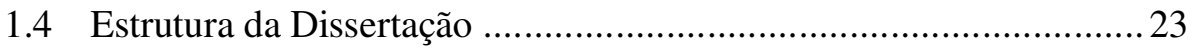

2 Panorama do Mercado Brasileiro de combustíveis ............................24

2.1 Da exploração do álcool ao surgimento do carro Flex-fuel ...............24

2.1.1 Histórico do álcool combustível no Brasil .................................24

2.1.2 A nova geração de motores Flex-fuel .........................................2 27

2.2 O preço dos combustíveis no Brasil em 2011 .....................................31

2.2.1 A formação dos preços dos combustíveis ...................................31

2.2.2 A competição entre etanol e gasolina ..........................................33

2.3 As vantagens ambientais do etanol frente à gasolina ..........................35

$3 \quad$ Referencial Teórico...................................................................................38

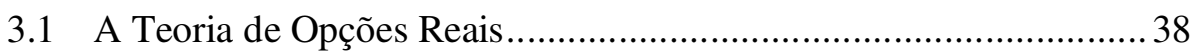

3.2 Processos estocásticos e Lema de Itô ................................................. 41

3.2.1 Processo de Markov ………………..................................... 42

3.2.2 Processo de Wiener ................................................................ 43

3.2.3 Movimento Browniano com drift ............................................... 44

3.2.4 Processo de Itô....................................................................... 45

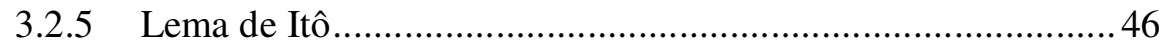

3.2.6 Movimento Geométrico Browniano ………………………........ 47

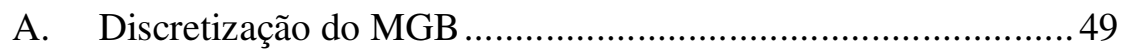

B. Estimação dos parâmetros do MGB ………………………....... 49

3.2.7 Modelo de Reversão à Média ................................................... 50

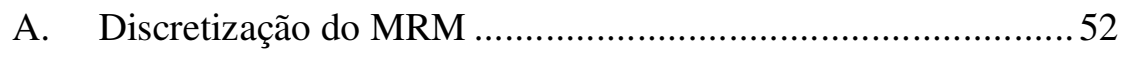

B. Estimativa de parâmetros do MRM ……………….....................54 
3.3 Determinação da Validade do Processo Estocástico

3.4 Simulação de Monte Carlo..............................................................59

4 Metodologia, aplicações e resultados .................................................62

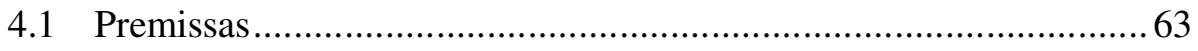

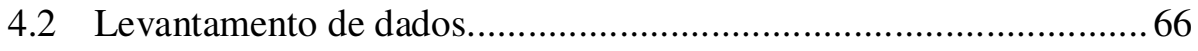

4.3 Limitações Metodológicas ....................................................... 68

4.4 Paridade entre os combustíveis nas regiões brasileiras .................. 68

4.5 A oportunidade associada ao carro Flex ….................................. 70

4.6 Determinação do Processo Estocástico que melhor se ajusta aos

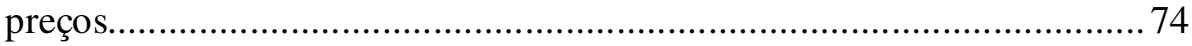

4.7 Aplicação do Modelo de Reversão à Média .................................. 79

4.8 Cálculo da opção de troca de insumo para o carro Flex ....................8 87

4.9 Análise dos resultados da Simulação.............................................. 89

4.10 Análise de Sensibilidade ................................................... 91

$5 \quad$ Conclusões e Perspectivas ...................................................................... 94

6 Referências Bibliográficas..........................................................97

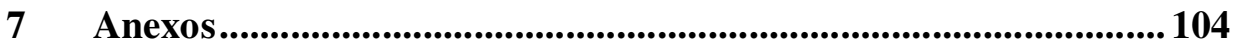

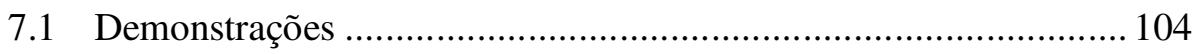

7.1.1 Propriedades estatísticas do MGB ........................................ 104

7.1.2 Propriedades estatísticas do MRM....................................... 107

7.1.3 Relação entre a velocidade de reversão e a meia vida do MRM

7.1.4 Ajustamento da média de longo do MRM prazo para um

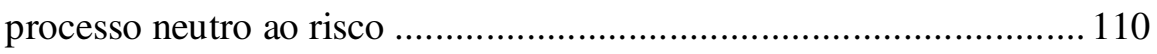

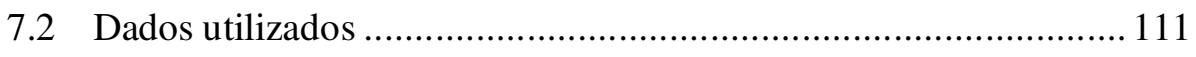

7.2.1 Valor do álcool e gasolina por região geográfica a preços de julho de 2001

7.2.2 Produção de etanol anidro e hidratado por estado 


\section{Lista de Figuras}

Figura 2.1: Participação de veículos leves por tipo de combustível nas vendas

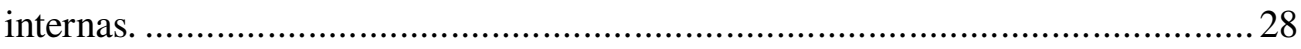

Figura 2.2: Consumo Brasileiro de etanol - em milhões de litros. ...................... 29

Figura 2.3: Produção Brasileira de etanol - em milhões de litros........................ 30

Figura 2.4: Produção interna de veículos leves por tipo de combustível.............. 31

Figura 3.1: TiposไClassificação de Opções Reais.............................................. 40

Figura 3.2: Funcionamento do processo para opção de troca de insumo.............. 40

Figura 3.3: Funcionamento da Simulação de Monte Carlo................................. 61

Figura 4.2: Preços médios do etanol e gasolina deflacionados pelo IGP-DI por região geográfica do Brasil,base jul-01...................................................... 67

Figura 4.3: Evolução dos preços do etanol ajustado e gasolina deflacionados na região Nordeste.

Figura 4.4: Evolução dos do etanol ajustado e gasolina deflacionados na região

Norte.

Figura 4.5: Evolução dos preços do etanol ajustado e gasolina deflacionados na região Centro-Oeste.

Figura 4.6: Evolução dos preços do etanol ajustado e gasolina deflacionados na região Sudeste.

Figura 4.7: Evolução dos preços do etanol ajustado e gasolina deflacionados na região Sul.

Figura 4.8: Resumo da escolha do processo estocástico.

Figura 4.9: Teste da razão da variância para o ln do preço do etanol por região geográfica.

Figura 4.10: Teste da razão da variância para o ln do preço da gasolina por região geográfica. 
Figura 4.12: Comportamento da média dos preços dos combustíveis simulados para a região Norte

Figura 4.13: Comportamento da média dos preços dos combustíveis simulados

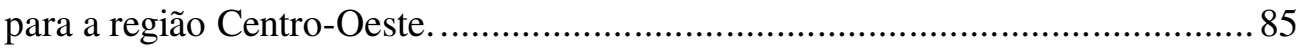

Figura 4.14: Comportamento da média dos preços dos combustíveis simulados para a região Sudeste.

Figura 4.15: Comportamento da média dos preços dos combustíveis simulados

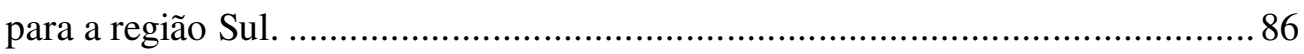

Figura 4.16: Análise de sensibilidade para a media de longo prazo do etanol......92 Figura 4.17: Análise de sensibilidade para a volatilidade do etanol e da gasolina. .93 


\section{Lista de Tabelas}

Tabela 2.1: Estrutura de Formação de Preço do etanol hidratado e gasolina C. ... 32

Tabela 3.1: Valores críticos assintóticos de teste $\tau$ da raiz unitária

(Dicker-Fuller) com uma constante e sem tendência temporal..........................58

Tabela 3.2: Valores críticos assintóticos de teste $\tau$ da raiz unitária

(Dickey-Fuller) com uma constante e tendência temporal..................................58

Tabela 4.1: Modelos de automóveis mais vendidos em 2011 (acumulado até

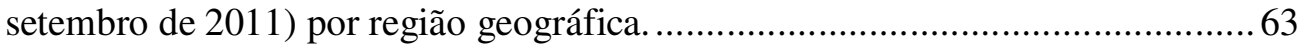

Tabela 4.2: Características dos veículos selecionados.......................................64 64

Tabela 4.3: Paridade média anual dos preços ao consumidor do etanol hidratado e gasolina $\mathrm{C}$.

Tabela 4.4: Resultados da regressão linear para o ln dos preços dos combustíveis por região geográfica.

Tabela 4.5: Parâmetros mensais para o MRM modelados para o preço do etanol e gasolina por região geográfica.

Tabela 4.6: Valor Presente dos custos do VW Gol movido somente a gasolina ou Flex por região geográfica.

Tabela 4.7: Valor Presente dos custos do Fiat Uno movido somente a gasolina ou Flex por região geográfica 


\section{Lista de Quadros}

Quadro 2.1: Problemas e Soluções para a exploração de cana............................... 36

Quadro 3.1: Analogia entre opções reais e financeiras..................................... 39

Fonte: Pessoa (2011) adaptado de Rigolon (1999)......................................... 39

Quadro 3.2: Quadro resumo para a estimação de parâmetros do MRM...............56

Quadro 4.1: Quadro resumo para a estimação de parâmetros do MRM...............81 


\section{Lista de Siglas e Abreviaturas}

CIDE: Contribuição Social de Intervenção no Domínio Econômico

CONFINS: Contribuição para o Financiamento da Seguridade Social

FC: $\quad$ Fluxo de Caixa

ICMS: $\quad$ Imposto sobre Circulação de Mercadorias e Serviços

IGP-DI: $\quad$ Índice Geral De Preços - Disponibilidade Interna

MGB: $\quad$ Movimento Geométrico Browniano

MRM: $\quad$ Movimento de Reversão à Média

PIS/PASEP: Programa de Integração Social

TOR: $\quad$ Teoria de Opções Reais

VPL: $\quad$ Valor Presente Líquido 